\title{
Per una caratterizzazione del giornale del primo Ottocento: la cronaca teatrale*
}

Il ricco quadro culturale della Milano napoleonica e della Restaurazione, è caratterizzato dallo sviluppo senza precedenti, e senza uguali all'interno della Penisola, della produzione giornalistica. Alle gazzette e ai periodici letterari di taglio settecentesco si aggiungono, soprattutto nel secondo quarto del secolo, giornali tecnico-scientifici e periodici di varietà in numero crescente. ${ }^{1}$ Nel 1805 si pubblicano a Milano due gazzette politiche, il "Corriere milanese" e il "Giornale italiano," un giornale di divulgazione tecnica, la "Biblioteca di campagna," una rivista di varietà, il "Corriere delle dame." Fra il 1810 e il 1825 nascono e muoiono giornali letterari anche insigni quali il "Poligrafo," gli "Annali di Scienze e lettere," il "Conciliatore," e si consolida la "Biblioteca italiana" e la serie dello "Spettatore." Dal 1825 al 1847 le riviste tecniche che vedono la luce sono almeno una decina e includono testate quali gli "Annali di statistica" e il "Politecnico," e al "Corriere delle dame" si vengono affiancando altri numerosi periodici d'intrattenimento e di teatro dalla vita piú o meno lunga e dai titoli variati ed immaginativi: l'"Eco," la "Fama," la "Moda," l'"Album," il "Pirata," il "Glissons n'appuyons pas," il "Cosmorama pittorico," il "Censore universale dei teatri," il "Corriere dei teatri," e altri. La ricca scena letteraria finirà con l'essere dominata dalla "Rivista europea," mentre l'informazione politica è affidata all'unico quotidiano, la "Gazzetta di Milano" che opera sotto l'egida del governo autriaco. Vivacissima inoltre la produzione annuale di almanacchi e strenne. ${ }^{2}$

A questa abbondanza e diversificazione di prodotti non corrisponde tuttavia né un'ampia circolazione, né la presenza di adeguate strutture redazionali e d'informazione. La tiratura media del periodico ottocentesco oscilla fra le 500 e le 800 copie - solo le gazzette politiche superano il migliaio, ${ }^{3}$ - e la compilazione è affidata in molti casi a una o due persone; si tratta di un'organizzazione artigianale con risorse economiche assai limitate. Fonte primaria della notizia è il periodico estero, soprattutto francese, ma anche inglese e tedesco, dal quale si traduce, si compendia, si adatta alle presunte 
esigenze del lettore italiano. E questo non solo per gli articoli politici, che sono sotto l'accurato controllo della censura e vengono tratti di solito dagli organi ufficiali dei governi in carica, ${ }^{4}$ ma anche per buona parte dei contenuti di varietà - narrativa, aneddotica, moda - e per l'informazione tecnica e scientifica. Si distingue già, tuttavia, l'attività in campo giornalistico di quegli intellettuali italiani che sono impegnati nella formazione di una nuova coscienza nazionale tramite la diffusione di precetti sociali, economici, civili: dal Romagnosi al Gioia, al gruppo del "Conciliatore," a Cesare e Ignazio Cantù, ai cugini Giuseppe e Defendente Sacchi, a Giacinto Battaglia, a Carlo Tenca, a tanti minori. 5 Ma, malgrado il loro infaticabile e prolifico operato, le centinaia di articoli dai contenuti piu disparati da loro contribuiti alle varie testate, la pagina del periodico milanese rimane ancora, nel complesso, dipendente dalle fonti straniere.

La mancanza di un vero rapporto con la realtà locale e la poca attualità dell'informazione sono particolarmente evidenti nella pagina politica. Le notizie riguardano quasi tutte l'estero e non sono mai recenti: i tempi di propagazione dell'informazione, già lunghi a causa della lentezza dei mezzi di trasporto dell'epoca, vengono praticamente raddoppiati per il lettore milanese. Egli infatti riceve la notizia, di solito, di seconda mano, solo dopo che i "fogli stranieri" sono arrivati a Milano e sono stati opportunamente "spogliati" e tradotti. ${ }^{\circ}$ Solo quando Milano o la Lombardia si trovano al centro di avvenimenti bellici di portata internazionale, il compilatore diventa anche redattore e interprete. ${ }^{7}$

Ma sono episodi isolati che non infirmano la norma, l'assenza cioè di una tradizione giornalistica locale. Lo stesso puo dirsi della cronaca cittadina, ridotta alla descrizione encomiastica di visite ufficiali di sovrani, nascite, battesimi, matrimoni illustri, conferimenti di cariche, inaugurazioni di accademie. Le direttive dei governi, in particolare di quello austriaco, furono ben precise: i giornali non dovevano comunicare notizie che potessero minimamente turbare la quiete e l'ordinato vivere dei sudditi. ${ }^{8}$ Persino i disastri di ordine naturale, alluvioni, terremoti, grandinate venivano cautamente ignorati o presentati con ottimismo e ritardo.

Anche con queste forti limitazioni sia economiche che politiche, il compilatore milanese veniva facendo il suo tirocinio giornalistico e veniva assumendo nuovi abiti linguistici. La fretta della compilazione, la minor selettività del destinatario, una programmatica ricerca di semplicità espressiva in accordo con la funzione divulgativa della stampa, ${ }^{9}$ contribuivano alla formazione di una prosa che cercava una sua connotazione al di fuori dei canoni della letteratura t:adizionale. E proprio la familiarità con i modelli giornalistici stranieri dovette favorire questo tentativo di emancipazione: la cronaca politica del periodico ottocentesco, ad esempio, riecheggiando gli 
originali francesi e inglesi, si distingue per chiarezza e linearità espositiva, favorita dal prevalere della paratassi e di periodi volutamente brevi. (Caratteristiche, queste, che non si sono necessariamente conservate nella successiva evoluzione linguistica dell'articolo politico italiano!). Analogamente, in altri tipi di articoli, si verranno mutuando dal piú evoluto modello straniero nuove categorie e nuove tendenze espressive, possibili parametri di scrittura giornalistica. ${ }^{10}$

Ma c'è un tipo di articolo che si distingue quale vistoso e originale contributo dei pubblicisti milanesi allo sviluppo del genere: la cronaca degli spettacoli. Essa accompagna - con una messe di recensioni, cronache, notiziari cittadini e esteri - la multiforme, vitale attività teatrale dell'epoca, e in particolare il melodramma, i grandi balli coreografici, i concerti, o accademie, vocali e strumentali.11 L'entusiastica partecipazione del pubblico e quindi l'esigenza di una informazione rapida e accurata, il ruolo preminente del teatro italiano anche in campo internazionale e lo stabilirsi di una industria dello spettacolo con legami fra le varie città europee e perfino extra-europee, ${ }^{12}$ l'aumento delle testate che vengono via via ad occuparsi di teatro, sono fattori che danno un impulso alla formazione di una classe di giornalisti specializzati e alla produzione in loco di articoli di vera attualità. $E$ in effetti se un paragone può essere fatto fra il pubblicista ottocentesco e il cronista moderno è proprio in questo settore. Allo scopo informativo dell'intervento, alla presenza di un rapporto diretto con un utente noto e disponibile e alle modalità di composizione, che sono alcuni dei requisiti di una produzione giornalistica, corrisponderà anche la presenza di alcuni elementi stilistici e lessicali che suggeriscono la formazione di un linguaggio settoriale.

Il teatro è il centro della vita artistica e mondana della città:

I divertimenti del Teatro alla Scala - dichiarava lo "Spettatore" nel 1818 - non sono meno necessari ai cittadini della pacifica Milano di quel che il fossero i giuochi del Circo ai cittadini di Roma Trionfatrice. - E proseguiva: - questo teatro è il centro di tutti i crocchi, il ridotto di tutta la classe agiata, il luogo che tutte le arti gareggiano nell'abbellire.13

Le rappresentazioni, e non solo nel Gran Teatro milanese, sono seguite con una costanza e un interesse che, superando il fatto di moda o di costume, raggiunge spesso momenti di fanatismo collettivo:

Chi conosce l'ampiezza del teatro La Fenice; chi sa immaginarselo affollatissimo di gente, delle cose musicali nel maggior numero non inesperte; chi è capace di raffigurarsi quella moltitudine irresistibilmente sedotta, trascinata a forza ad interrompere gli stessi piaceri suoi, per prorompere in grida altissime di gioia e di plauso; e tut to questo per tre 
sere consecutive, senza discordia di opinioni, anzi con sempre crescente entusiasmo, quegli potrà da solo giudicare quale sia stato il suo trionfo. ${ }^{1+}$

Non sorprende quindi se, rispondendo alle esigenze del pubblico, ogni tipo di periodico, ad eccezione di alcune riviste tecniche, dedicherà ampio spazio alla cronaca degli spettacoli, la pagina certamente piú letta e piú attesa. La cronaca, presente fin dall'inizio del secolo sia nei periodici d'informazione politica che in quelli letterari, sia nel "Corriere delle dame," subisce un aumento notevolissimo dopo il 1828-1830. Sono questi gli anni che vedono il proliferare della stampa periodica, soprattutto quella d'intrattenimento, e la nascita di testate dedicate esclusivamente al teatro. Ė interessante notare come in molti casi, giornali nati con intenti genericamente divulgativi, quali ad esempio l'"Eco" o la "Fama," debbano aumentare il contenuto teatrale - spesso introducendo Appendici apposite - probabilmente per riuscire a sopravvivere alla concorrenza, e questo a tutto scapito degli altri generi: narrativa, aneddotica, cognizioni utili, informazioni varie. Le riviste piú impegnate, in particolare la "Rivista europea," si occuperanno del teatro soprattutto come fatto letterario e artistico, trascurando il lato cronachistico. Anche gli almanacchi e le strenne sono presenti con alcune testate specializzate, fra le piú note l'"Almanacco del Teatro alla Scala" e la "Strenna teatrale europea." 15

La cronaca teatrale assume quindi, nel quadro generale del giornalismo italiano dell'epoca, un' importanza particolare: non solo essa è la parte piú genuinamente originale nella composizione, ma è anche l'unica attestazione costante di cronaca cittadina, sia pure settoriale. Essa è inoltre il settore nel quale si rivela maggiormente l'esigenza per l'attualità e la rapidità dell'informazione. Di questa esigenza si fa portavoce il giornalista stesso: nella continua ricerca di "accuratezza nel rintracciar le notizie ... e sollecitudine nel comunicarle"; 10 nella organizzazione di una rete di contatti e scambi con altre città, tramite "corrispondenti" o "postiglioni" incaricati di trasmettere notizie "genuine e guarentite . . . con personale testimonianza"; 17 nelle modalità stesse della composizione: rapida stesura di resoconti di spettacoli locali, "genuini recentissimi ragguagli del qui accaduto." 18 Si rivela cosí, sempre nei limiti di una realtà ottocentesca e italiana, la presenza di professionisti dell'informazione, in possesso di strutture tecniche già abbastanza sviluppate.

La consapevolezza dell'originalità del proprio prodotto spinge talvolta il compilatore del foglio teatrale a disprezzare altri tipi di pubblicazioni periodiche, proprio in quanto i loro contenuti mancano di attualità e dipendono ancora dai deprecati modelli stranieri: 
Da me non avrete, né potete avere che relazioni teatrali esposte come la natura le detta - dice il compilatore del "Corriere dei teatri" nel suo annuale saluto al lettore. - Chiedere non potete ... che siano da me spogliati i fogli francesi per trattenervi con istorielle, con aneddoti di questo o dei passati secoli. Se queste coserelle non le avete sentite da fanciulli, le incontrate quotidianamente in tanti altri fogli, che per non farvele perdere di vista, ve le ripetono qualche volta, e piu spesso ancora se le copiano a vicenda. ${ }^{19}$

Il cronista teatrale potrà inoltre esercitare una valida missione, soprattutto se saprà conservare indipendenza ed etica professionale. Guai ai "signori giornalisti e gazzettieri troppo affezionati al Catalogo delle sottoscrizioni" e sempre pronti a "conformarsi al voto del pubblico"; chè, al contrario, "uffizio, anzi dovere di chi ardisce di propagare con le stampe il proprio parere intorno al merito o ai difetti di una qualunque produzione teatrale, sta appunto nel far coraggiosamente toccare con mano al Pubblico l'erroneità o l'inconsistenza di molti dei suoi giudizi."20 E guai a coloro invece che si lasciano trasportare da simpatie o "passioni" personali: "l'abiezione e lo sconforto che colpisce la missione giornalistica teatrale non deriva dal coprire di encomio il vero merito teatrale, ma sibbene di farlo scopo delle proprie passioni." 21

Celerità, accuratezza e obiettività nella comunicazione, formazione del gusto, critica costruttiva delle carenze del genere, ecco le caratteristiche ideali del giornalismo teatrale dell'epoca. L'articolo teatrale infatti, non è solo il réportage del più diffuso passatempo dell'epoca: il critico, a volte anche quello che scrive su giornali eminentemente divulgativi, si fa portavoce di esigenze di riforma. Se da un lato, per esempio, l'immensa popolarità del genere e i successi degli artisti italiani all'estero vengono esaltati come unica, ultima sorgente di gloria nazionale, "nella convinzione comune - per usare le parole del "Corriere delle dame" - che la bella Italia puo tuttora portare il vanto d'esser madre di Classici maestri e mantenersi con ragione il primato nella bellissima fra le arti belle"22 dall'altro c'è chi scorge in questo patriottismo teatrale la ricerca di un facile successo editoriale, e lo denucia aspramente:

Si diffidi degli impiastrafogli - scrive la "Rivista europea" - che sono sempre pronti a propalare i trionfi e le vicende dei cantanti, ballerini, impresari, coreografi, saltatori, giullari, ciurmatori e simile razza di gente

perché questi mantengono in vita i loro giornali ... [Essi] hanno sempre per bocca l'amor di patria. Per essi l'amor di patria sta nel lodare la sveltezza, o la forza delle gambe di questo ballerino, la spigliatezza e leggiadria di quella. ..."23

La parte dedicata alle polemiche sul genere e alla denucia delle sue carenze è tuttavia quasi trascurabile di fronte all'abbondanza di 
Bollettini o Notiziari teatrali che tengono informato il pubblico su tutta la vasta gamma di attività in corso, e delle attesissime recensioni degli spettacoli. Il teatro accompagna infatti ogni evento e ogni ricorrenza sociale in città come in provincia: non c'è fiera, non c'è festeggiamento ufficiale, non c'è carnevale che non richieda l'allestimento di una stagione piú o meno ricca, di rappresentazioni teatrali. ${ }^{24}$ La passione per il teatro accumuna gli intenditori di città e di provincia, e i giornali, non insensibili alle esigenze dei loro lettori, si fan premura di tenerli al corrente sulle stagioni teatrali degli altri centri. Fatto tanto piú notevole quando si pensi alla mancanza di informazione negli altri settori, soprattutto durante la Restaurazione: la notizia teatrale è cosí spesso l'unica a portare al pubblico milanese echi della vita di altre città italiane. Diventano sempre piú abbondanti, con il passare degli anni, anche le notizie da varie città europee, città che van prendendo "l'abitudine di ricrearsi . . . col canto italiano" e "dall'abitudine imparano ad essere da stagione a stagione sempre piú esigenti." 26

Ma è il pubblico italiano, di appassionati cultori, che è il protagonista di molte di queste pagine di cronaca teatrale. E, soprattutto quello che frequenta i teatri milanesi, un pubblico d'intenditori a volte "poco compassionevole," 27 a volte "prevenuto e difficile," 28 ma piu spesso prono a entusiasmo, fanatismo, furore. ${ }^{29}$ Il cronista fissa talvolta l'occhio con commozione immemore su una platea particolare:

Bella a vedersi era quella sala teatrale fitta di spettatori, fra i quali un gran numero si scorgeva di gentili ed avvenenti giovinette, sul cui volto brilla il fiore della vita e quell'animato sguardo, che alcune volte va a colpire i cuori che non san difendersi da improvvisa impressione ché tale è la magia degli occhi che giungono ad eccitare una passione, la quale si può bensí vincere, ma non mai obliare si puo né il volto né lo sguardo che l'han fatta nascere. Ma dove scorre la penna?30

A volte descrive con assonanza stilistica un crescendo di consenso:

. . Ma la delicatezza di gusto pel bello musicale esiste nel nostro uditorio, e questo finissimo senso si stanco finalmente di sonnacchiare; ed eccolo risvegliato e sereno nell'atto secondo, che scoppia in fragorosi applausi alla barcarola . . . che diventa entusiasta al duetto della buffa con buffo, e ne domanda avidamente la replica, e tanto piu si esalta nell'ottenerla, che si inebbria a quelle ultime soavissime note ...31

o registra accuratamente un successo strepitoso:

le acclamazioni universali si prolungarono con incremento di vigore dopo calato il sipario, ed a queste acclamazioni apparirono sul proscenio $i$ tre primi cantanti; lo strepito non cesso per questa apparizione, anzi 
crebbe, ed i tre cantanti ricomparvero; né di ciò soddisfatta mostrossi la pubblica effervescenza, ma si rinnovo anzi per la terza volta, e per la terza volta ebbe i suoi cantanti sul proscenio . . . ."32

\section{osserva un comportamento compassato:}

Non so per qual ragione, quest'anno il pubblico [del Gran Teatro] è freddo, tranquillo, indifferente, di rado si scuote, e non muove le mani ad applaudire, se non quando il prestigio dello spettacolo è tale da vincere ogni apatia. Forse che ha sciupato interamente l'entusiasmo nelle ovazioni e nelle gare degli anni passati, forse anche vuole espiare con una condotta esemplare quei frenetici trasporti, a cui troppo facilmente si lasciava andare: il fatto è che gli applausi suonan rari e misurati. ${ }^{33}$

\section{O denuncia con affettuosa ironia l'arbitrarietà di certi giudizi popolari:}

Pubblico mio, tu hai trovato perfetto, divino, magnifico quel tale o tal' altro lavoro musicale, quando non è che una cosa poco piú che mediocre; Pubblico mio, tu hai soffocato colle tue maledizioni uno spartito che pur avea del buono; Pubblico mio, tu applaudi ai gorgheggi, e alle scimmiotterie di un Cantante che meglio andrebber fischiate. . ." 34

o condanna le "macchinazioni di pochi malevoli" che tentano invano di influenzare le sane reazioni di un pubblico "giusto":

Vive sotto ogni cielo un'infesta razza di notturne strigi, il cui ululato, pari al lamentar degli Alcioni, si piace di pronosticar sempre tempeste e naufragi. Fra le malaugurose strida di sitenebrosi augelli comparirono su queste scene gli attori. .. Ma in faccia al vero merito ed al cospetto di un pubblico giusto, rifuggono le macchinazioni di pochi malevoli, come le nottole all'apparir della luce." 35

Gli eroi di questa epica dello spettacolo sono tuttavia gli artisti, e in particolare gli interpreti, i divi del canto, della danza, degli strumenti. Cosí l'entusiasmo per Paganini è senza confini:

Esecutore sommo a un tempo nel grave, nell'allegro, nel delicato, nell'espressivo, nel robusto, nel difficile, e nel complicatissimo, egli tocca quella meta, oltre la quale è impossibile a umano ingegno il procedere innanzi ...36

quello per la Malibran - come per tanti altri astri del palcoscenico - apertamente enfatico:

Ella venne, ed ella si produsse nell'Otello, e fu come l'Angiolo del conforto e della gioja, come la riparatrice di tanti danni, e delle tante noje sofferte, come la ristoratrice delle nostre povere orecchie: con lei si ammutirono le 
paure dei timorosi, si assicurarono gli animi degli increduli, e fino gli indifferenti, quelli che non aprono mai il loro cuore, né alla speranza né al gaudio, si sentirono scossi, agitati, e trovarono che la musica sulle labbra della Malibran aveva qualcosa di prodigioso . . . ${ }^{37}$

quello per la Pasta - la febbre pastomaniaca - ispira "frasari da secentista":

Noi, ben lontani dal disapprovare quell'omaggio, che giustamente si deve al merito della Pasta, confessiamo anzi di essere ammiratori di questa incomparabile artista, senza pero essere predominati da quella pastomania, la quale ha fatto dare parecchi in tali stranezze, che provano una manifesta alterazione di mente. - Tra gli altri un febbricitante dell'Adige, in un Dialogo, non ha guari dettato nel bollor della febbre, con un frasario da secentista ebbe a chiamare la Pasta, sole del canto e dell'azione drammatica; fiore composto di tutte le eccellenze del mondo; quintessenza delle donne." 38

I giornali contribuiscono cosí alla formazione del culto per i divi. E se l'entusiasmo popolare per l'uomo eccezionale non è certo un fatto nuovo, quello che è nuovo, o comunque assume nuove proporzioni è il ruolo della stampa periodica nel confermare e incoraggiare certe tendenze (un ruolo già affidato alla stampa del primo Ottocento dalla consapevole politica del consenso del piu spettacolare divo dell'epoca, Napoleone. ${ }^{39}$ )

L'articolo teatrale si presenta dunque come uno dei settori piú vitali del periodico italiano dell'Ottocento: come momento nello sviluppo di un genere giornalistico esso si distingue per l'intento informativo, l'attualità della notizia, la relativa ampiezza della diffusione, la gestione da parte di "professionisti" dell'informazione; come documento storico esso riflette interessi, voghe, problematiche della società dell'epoca, attraverso la ricca, fervorosa descrizione del mondo dello spettacolo e dei suoi interpreti. Per questa descrizione il cronista fa ricorso ad una varietà di registri espressivi e di soluzioni linguistiche, animato da esigenze che - pur in una chiara configurazione ottocentesca - possono definirsi giornalistiche.

Non è intento di queste pagine il provare se a queste esigenze corrisponda lo stabilizzarsi o comunque l'apparire di una scrittura giornalistica; 40 sarà tuttavia possibile suggerire la presenza di alcuni fattori che di tale scrittura sono prerequisiti. Piu di altri prodotti della pubblicistica dell'epoca, la cronaca teatrale è destinata al rapido consumo di un pubblico vasto ed eterogeneo; il pubblico stesso, fra l'altro, che ha assistito allo spettacolo teatrale e che potrà quindi facilmente identificarsi nel messaggio recepito. Si tratta ovviamente di "vastità" ed "eterogeneità" tutte relative: il periodico ottocentesco ha, come si è visto, una circolazione cosí ristretta da non potersi certo considerare un mezzo di comunicazione di massa. 
Ma entro questi limiti inevitabilit1 è proprio la cronaca teatrale che si distingue per la popolarità e per l'alto indice di gradimento, se si deve giudicare dall'aumento della sua presenza nell'arco di tempo da noi esaminato. E il linguaggio, malgrado il legame strettissimo con la tradizione letteraria, è abbastanza innovativo, tende a chiarezza espositiva (soprattutto a livello sintattico), è incline alla codificazione di speciali formule tecnico-settoriali.

Emancipato o comunque indipendente dai modelli stranieri che ancora dominano nel campo della notizia politica, della narrativa e della divulgazione - l'articolo teatrale è prodotto originale, non traduzione o rifacimento; le scelte linguistiche dipendono perciò, piú che in altri generi di articoli, dal giornalista stesso e quindi dalla testata che lo ospita. Fattore determinante per la tecnica espositiva sembra pero, piú che la testata in esame la tipologia dell'intervento: il "notiziario teatrale" si differenzia infatti dalla critica o recensione di un'opera e della sua esecuzione. Il primo - eminentemente informativo - tende all'esposizione lineare e concisa di materiale cronachistico: si tratta di ragguagli spesso assai brevi sui teatri stranieri o sulle attività degli artisti, che danno luogo talvolta a rubriche dai titoli significativi, quali le Notizie telegrafiche dalle provincie, del "Corriere dei teatri," o le Varietà teatrali straniere della "Moda," o l'Un po' di tutto del "Pirata." La seconda, spesso assai ampia — non sono rare le recensioni a piú puntate - ricorre spesso a moduli della saggistica tradizionale e a strutture piú complesse. All'interno della recensione stessa, poi, quello che sembra condizionare le scelte linguistiche è l'atteggiamento critico, positivo o negativo, assunto dal giornalista: i resoconti di successi strepitosi, l'esaltazione dell'eroe o dell'eroina del momento, la descrizione delle qualità estetiche della musica, del canto, della danza, richiedono l'uso di ogni possibile supporto esornativo con un generale prevalere di intonazione aulica; la constatazione di un insuccesso, o la stroncatura piú o meno feroce dello spettacolo o degli interpreti si appoggiano invece spesso - soprattutto per il predominare del discorso satirico - ai modi del registro brillante. Comune a tutte le testate e a tutti i tipi di articoli è comunque la predilizione per gli artifici retorici quali l'iterazione, la gradazione, l'apostrofe, l'interrogazione, la struttura ternaria e, specialmente, l'iperbole. Sia il brano aulico che quello brillante pullulano di traslati, e alla metafora tradizionale si aggiunge talvolta quella innovativa. Inoltre, questa lettura preliminare, non sembra fornire elementi che suggeriscano chiaramente uno sviluppo diacronico del genere nei quarantasette anni presi in esame: si avverte infatti un ripetersi e avvicendarsi delle stesse soluzioni stilistiche. 
Se già la lettura dei brani precedentemente citati può esser servita a dare un'impressione dell'atmosfera linguistica che si sta cercando di definire, una campionatura più sistematica potrà avvalorare alcune delle ipotesi formulate. Il registro aulico, che prevale come si è detto nelle recensioni, è facilmente percepibile anche nelle brevi selezioni su cui ci siamo soffermati: nel generale esibizionismo verbale, nell'ampio giro della frase ad andamento narrativo - "Bella era a vedersi quella sala teatrale. .."; "Vive sotto ogni cielo..."; "Ella venne, ed Ella si produsse ..." - nei cultismi lessicali "notturne strigi" - nel "frasario da secentista" (segnalato del resto dal giornalista milanese). Ma, a confermare certe tendenze potrà servire un breve brano di prosa esornativa del "Poligrafo," classicisticamente culto:

Dal coro de' giovanetti e delle donzelle di questo patrio istituto, tenuto è in serbo il fuoco sacro di quell'arte divina, che infiammo il petto de' grandi compositori italiani; laonde mercè della sollecitudine e del sano gusto che presiedono all'ammaestramento di questa scuola, vedremo ben presto uscire dal seno suggetti abilissimi sí nel canto come nel suono, i quali cingeranno di nuovo sulla fronte della nostra Erato quella corona, di cui gli odierni satelliti delle sette d'oltremonte si studiano a spogliarla . . . 2

o alcune righe del "Censore universale dei teatri" ricche di inversioni:

Non hanno le nostre scene un soggetto che invidiare non debba a questo il vantaggio della figura: un armonico e propriamente accademico aggregato di maschili forme, una per il regolare e ben pronunziato disegno de' suoi lineamenti interessante fisionomia compongono la sua bella persona . . .43

o il sostenuto paragone naturalistico dell"'Eco," nella favorita struttura ternaria;

Quella composizione può essere paragonata ad un ameno giardino; fiori spandenti soave profumo vi abbondano, limpido ruscello con dolce mormorio vi serpeggia, verdeggianti praticelli vi si alternano a deliziosi viali di piante; il tutto insieme forma un incanto che v'infonde nell'anima un delizioso sentimento di contentezza . . .44

o l'efficace triplice ripetizione di nero nel ritratto di una giovane cantante:

I suoi neri, folti capegli davano risalto alla candidezza della di lei fronte; e sotto le nerissime ciglia, due grandi occhi neri brillavano come raggi del sole d'Italia." 45

Ternaria è anche l'apostrofe satirica "Pubblico mio. .. Pubblico mio. . . Pubblico mio ..." segnalata precedentemente; e, abilmente 
orchestrata in due costrutti ternari con ripetizione dell'ultimo termine, una delle tante lodi di artisti famosi:

fra gli odierni tenori una delle primarie stelle, il desiderio di tutti gli impresari, il cantante dalla voce simpatica e dai modi gentili, il cantante che ben a ragione ora si merita il nome di animatissimo egregio attore, fu per la terza volta l'ornamento di quel teatro, il lustro di quelle scene, l'amore de' viennesi, l'amore di tutti coloro che in un'arte qualunque curano e cercano il bello. ${ }^{40}$

Le terne abbondano sia nell'enumerazione rapida - la Gazza ladra "piace, commuove, elettrizza" - sia nella ricca aggettivazione voce "bella, estesa omogenea" o "piena, flessibile, dolcemente insinuante," pubblico "freddo, tranquillo, indifferente."

All'aulicità del costrutto si accompagna il gusto per l'iperbole, tanto nei concetti - il canto "è il più bel dono concesso dal cielo agli uomini," la Ferlotti canta cosí bene che "il cantar meglio non è nella natura umana," quella francese è "la prima scuola di danza dell'universo," - quanto nelle scelte semantiche - "inesprimibile energia dell'azione," "merito trascendente," l'"unica Pallerini," l'"esaltatissima Tosi." Anche le numerose perifrasi e antonomasie tendono all'iperbolico: lo scrittore tragico segue "le sublimi vie del coturno," Rossini è "il sublime cantore di Pesaro" e la Pasta "la Corinna del canto." Iperbolico o generalmente enfatico è il tono di tante descrizioni; ecco la Essler, una grande ballerina dall'effetto miracoloso:

Pare veramente che nelle sue fibre corra una vita diversa della comune ... Il miracolo è naturale. V'ha qualcosa di cosí magnetico in quei movimenti, un fremito quasi che da quelle forme armoniche e perfette si diffonde per tutto il teatro, che ognuno sente raddoppiato in sé il sentimento della vita . . .47

e madamigella Angelini, "maestra nell'arte pantomimica" e nella seduzione:

. hanno i suoi gesti una dominante espressione ed i suoi piedi un seducente incanto che attrae, che seduce. . . . Nella sua maniera di ballare v'è leggerezza indescrivibile, ed esattissima precisione di tempo ... accoppiata alle grazie ed alle proporzioni pittoriche del suo corpo decentemente velato $\ldots .8$

ed ecco l'insuperabile coreografo di grandi balli, Salvatore Viganò:

Viganò si è colla Vestale collocato in cima alla difficilissima arte sua ch'egli ha condotta per nostro avviso, all'estremo grado di perfezione. Noi osiamo 
sfidarlo a superare sè medesimo, se è possibile, giacché non ha egli né emuli né rivali da poter vincere $\ldots 4^{\circ}$

ecco, infine, uno dei tanti furori di pubblico:

la seconda sera fu propriamente quella che assicuro allo spettacolo l'incontro del furore; furore che risuonò negli applausi più veementi dei primi, che alla fine di tutti i pezzi volle sul proscenio i cantanti; furore che conservò l'entusiasmo in tutti gli uditori vivissimo nelle recite successive, ed anzi lo fece tanto ascendere alla quinta e alla sesta replica da non potere andare piu in alto." 50

Il tessuto eminentemente retorico di queste pagine si rivela inoltre nell'ampio uso del traslato: nei brani ad intonazione aulica le metafore sono di preferenza quelle mutuate dal nostro patrimonio letterario-poetico. Abbondano le variazioni sul tema della luce, del fuoco, del sole: i successi sono luminosi; gli artisti brillano, riscaldano col loro calore, irradiano, cosí come le tragedie agghiacciano, le brine onde s'imbianca il capo di un rinomato maestro spengono la sacra fiamma dell'ispirazione, e il bollor della febbre pastomaniaca ispira in un febbricitante le già citate "metafore da secentista." Altrettanto abbondanti i temi marino-meteriologici: "Nel comune naufragio degli spettacoli il nostro poté giungere in porto, dopo piccola burrasca e qualche avaria"; anche gli applausi "Scoppiano in una vera procella" e Donizetti "urtò in fortissimo scolgio alla Scala ... per cui ebbe a precipitar negli abissi."

Ma in una buona parte delle recensioni il giornalista teatrale abbandona il registro aulico, pur cosí suasivo, a favore di quello brillante; si tratta, come si è detto, soprattutto - ma non esclusivamente - di brani a intonazione satirica e nei quali si fa sfoggio di abilità verbale. Le metafore in questi casi sono meno legate alla tradizione letteraria e attingono liberamente a lessici di altri settori. Una recitazione troppo enfatica è una palla di fucile, un bombardamento, una cannonata; le notizie provenienti da altri giornali sono "mercanzia esposta come fu venduta" e il cronista lascia ai "dilettanti la cura di ascegliere a piacer loro, perché il piú delle volte la merce alterata è merce guastata"; un malaugurato cantante "operò dei salti mortali colla gola a rischio di ammazzarsi ad ogni volta" e un nuovo melodramma "mori di apoplettico colpo, e pace alle ceneri sue." La celebratissima Pasta è ispirata da scintilla elettrica, e la Essler ha qulcosa di magnetico nei suoi movimenti. La crescente fortuna di fiasco - "parola tecnica alla quale non è oggi piú unita nessuna idea disonorante," si spiega già nel $1806^{51}$ - suggerisce una ripetuta metafora alimentare che ha lo slancio di un brano pubblicitario: 
I fiaschetti di Firenze pieni di Monte-pulciano, o di aleatico, si bevono in Livorno come altrove; ma siccome si trovano troppo piccoli, chi ne ama di piú grandi a forma di damigiane, vada alla fabbrica del sig. Rendini, compositore del ballo intitolato il Paggio di Leicester, che ne vende a buon prezzo, ma vuoti affatto. 52

Nel "brillante" anche la tecnica espositiva vuole essere diversa. Cosí accanto al brano magniloquente trova posto il pezzo volutamente svelto e spigliato, a volte compendioso. Qui, la ricerca di effet to viene affidata all'incisività dell'esposto, al rapido succedersi delle unità di contenuto, alla frequenza delle frasi nominali:

Dopo la Lucia comparvero in iscena i Puritani, ed ho il piacere di annunziarvi un secondo furore. Eccovene il dettaglio. Grande affluenza di popolo al teatro, perché i presenti Puritani non sono quelli impasticciati dal Pugni, che abbiamo avuto altra volta. Applausi vivissimi all'introduzione. Applaudita la cavatina di Riccardo (Superchi). Deciso entusiasmo il duetto della Strepponi, e Giorgio Ronconi con due chiamate. Fracasso inaudito alla sortita di Moriani, che termino a fanatismo. Incontro assoluto la Polacca della Strepponi con tre chiamate: cosí il finale: applausi immensi in special modo al largo, con due chiamate a tutta la compagnia alla fine, appena calata la tela. ${ }^{53}$

Anche il concentrarsi di tecnicismi (duetto, cavatina, polacca, largo) e di traslati settoriali ( chiamata, incontro, fanatismo, furore), e la presenza di vocaboli mutuati dal parlato o comunque dal non letterario (impasticciati, fracasso) contribuiscono alla caratterizzazione di una prosa quasi gergale.

L'esposizione rapida a ritmo sostenuto è presente in moltissimi brani cronachistici, quali un resoconto dal Teatro Re sulla prima dell'Italiana in Algeri:

Un intreccio piacevole ed inverosimile; una musica, che dalla sinfonia procede fino al fine con sempre crescente amenissima armonia; sette attori, uno de' quali è mediocre e tre sono ot timi; finalmente un'orchestra ben diretta formano quattro requisiti, che assicurarono il buon esito di questa opera ....54

o quello di una rappresentazione periferica - a Udine - dei Puritani:

Ne daremo prima la storia. - Atto primo. Introduzione dei cori, silenzio. Cavatina del basso Guscetti, applaudita con due chiamate. Quartetto, sortita del tenore Storti, a meraviglia, con applausi pure alla valente Boldrini. Polacca d'Elvira, benissimo. Finale, silenzio - Atto secondo. Introduzione del basso Torre, qualche applauso. Rondo della Boldrini, un deciso fanatismo .... (ecc.)."55 
E l'incisività diventa la norma nei Bollettini o Notiziari teatrali che compaiono sempre piú frequentemente sulle varie testate; in una rassegna di spettacoli alla Scala (che culmina nella stroncatura lapidaria, zero piú zero fa zero):

Agbar Gran Mogol del sig. Gioja: la composizione è poca cosa; vi si trovano delle bellezze qua e là sparse. Buona scelta di musica, ma il piú delle volte male applicata.

La Cenerentola del sig. Bertini per secondo ballo: Le dolci ricordazioni del dramma e della musica di Rossini hanno fatto gustare quest'azione pantomimica.

Il vascello d'occidente Melodramma di . . . ec.: Non fu possibile di riprodurlo dopo la prima sera, nella quale un urlar sí violento ed un fischiar si forte cagiono il naufragio del vascello. Per buona sorte l'equipaggio, ció nonostante a gran stento, si salvo.

Il finto feudatario del sig. Blasis per secondo ballo: zero piú zero fa zeroso

in una serie di "brevissime" da città italiane e straniere che la "Gazzetta di Milano" allinea di solito in ordine alfabetico:

MANTOVA - Fortunato incontro ottenne su questa scena la Regina di Galconda nella quale vi cantano con plauso: la Manzocchi, il Bonafos, il Paterni e il Profeti.

MODENA - Ottimo successo ebbe qui il Belisario colla Novello, Pasini, il Fornasari e l'Albertazzi. L'orchestra fu al solito degna d'encomio.

NUOVA ORLEANS - Questo teatro di San Carlo soggiacque ad un grande incendio, che lo ridusse in cenere" 57

nell'Un po' di tutto del "Pirata," in cui le notizie compaiono di seguito, separate solo da un trattino:

L'Olivo e Pasquale, succeduto a Casalmonferrato allo Scaramuccia, piacque e piacerà sempre piú. Si attende un'opera seria. - Il celebre Donizetti è il capo della Commissione della Musica Italiana istituita a Parigi per l'innalzamento della statua a Rossini. - Del Corrado che il maestro Federico Ricci deve esporre sulle scene italiane di Parigi e che canteranno la signora Grisi, la signora Marietta Brambilla, Mario e Giorgio Ronconi, non è rimasta che la musica: il poema è tutto cambia to.

- Piacque a Zarra il Columella con la signora Mazza, il Tasca e i Bostoggi. - (ecc). 58

All'interno dei diversi tipi di interventi che si è cercato di esemplificare - critiche, recensioni, cronache, notiziari - e nelle diverse soluzioni stilistiche, il vocabolario appare piuttosto ristretto e ripetitivo, caratterizzato come si è detto dall'avvicinamento di 
tecnicismi lessicali e stilemi retorici mutuati da altri sottocodici. Bastino alcuni esempi tratti dai termini piu ricorrenti: fra le parole tecniche, basso, basso-cantante, buffo-cantante, contralto, soprano, tenore, duetto, recitativo, ecc.; fra quelle usate tecnicamente o caricate di valori connotativi conferenti un valore specifico: aria, azione, stagione, amatore, "abituato," concorso, incontro, ecc. Non abbondanti i neologismi, spesso rappresentati da usi particolari o metaforici di parole particolari, si pensi ad esempio all'uso, già indicato, di fiasco e della neo-formazione fiascheggiare. Debutto e debuttante sono sentiti come francesismi ancora nel 1830 ("che dirò la debutante perchè non lo so dire in una parola italiana" spiega l'"Eco"s9). Ma i forestierismi sono assai rari in un campo che ha visto invece la diffusione all'estero della terminologia musicale italiana. Alcuni termini sembrano peculiari del linguaggio teatrale del momento, cosí dilettante per amatore/intenditore, e corrispondente per impresario. Concorso e furore vengono usati in senso assoluto per indicare concorso o furore di pubblico. Gli stessi giornalisti dell'epoca sono consci dell'abuso di un linguaggio pieno di "espressioni" e "motti" da iniziati, e della volubilità dei traslati alla moda nello "stile delle relazioni teatrali": si senta una denuncia del "Corriere delle dame," in calce a dei Brevi estratti da giornali italiani:

Vedranno i nostri lettori da queste brevi notizie quale cambiamento siasi introdotto nello stile delle relazioni teatrali. - Una volta usavano le espressioni a gonfie vele, con prosperi venti, oppure a timone spezzato con venti aquilonari: il ballo per esempio naufragava: il compositore restava annegato sebbene lo spettacolo terminasse con un incendio come quello di Tauris del signor Rigali. Si usava dire l'opera ha fatto fiasco o fiascaccio o fiaschetto, oppure ha fatto furore. Ora i furori si sono lasciati alla senavera e vi si è sostituito il motto a cielo: la parola fiasco non si trovó registrata sul vocabolario e i puristi modigrafi vi sostituirono a terra. Parimenti vuole la moda che i cantanti si chiamino astri, o stelle, soli, lune, mezze lune, ecc. ecc. Exempli gratia, la prima donna $X$ fu la luna dello spettacolo. . .60

Caratteristiche formali e di contenuti, modalità di composizione e di uso, autorizzano dunque a considerare l'articolo teatrale come la parte più genuinamente giornalistica del nostro periodico. Il teatro è seguito con entusiasmo dal pubblico, coinvolge gli interessi di una classe di operatori economici, alimenta la costruzione del culto del divo. E i giornali se ne impossessano, ne seguono le vicende, ne favoriscono la popolarità. Nel quadro generale dell'informazione dell'epoca, imbavagliata nel campo politico e bandita da quello della cronaca locale, è solo l'articolo teatrale che può svilupparsi liberamente e cercare modi espressivi che rispondano alle esigenze sue e del suo lettore. Si tratta di uno sviluppo settoriale, ben inteso, che non indica necessariamente l'affermarsi di una scrittura giornalistica in senso lato. Pure, i caratteri generali di 
questa prosa permettono un accostamento - che meriterà una verifica sistematica - con un tipo di linguaggio giornalistico odierno, quello della cronaca sportiva. La cronaca teatrale ottocentesca, come la cronaca sportiva odierna, puó presentarsi in abiti curiali, o tendere all'effetto brillante, o condensare le notizie in brevi rassegne. Ambedue arricchiscono e nobilitano i loro contenuti con forme mutuate da generi di maggior prestigio, soprattutto quello letterario; ambedue si appoggiano all'uso dei traslati piú spericolati nella ricerca di una massima spettacolarità. Ambedue assolvono con efficacia un compito divulgativo e informativo rispondendo alle esigenze emotive di un pubblico particolare.

\section{University of British Columbia}

\section{NOTE}

*Queste pagine si configurano nel quadro di un ampio studio sulla stampa periodica milanese, condotto dall'autrice e da due colleghi nel corso di vari anni. Esse precedono di poco la pubblicazione di una serie di volumi di consultazione, che di questo studio sono un primo frutto: Stefania De Stefanis Ciccone, Illaria Bisceglia Bonomi, Andrea Masini, La stampa periodica milanese della prima metà dell'Ottocento: Testi e Concordanze (Pisa, 1982).

1 Gli studi piu aggiornati e autorevoli sul giornalismo dell'epoca sono quelli raccolti nella Storia della stampa italiana a cura di V. Castronovo e N. Tranfaglia, e in particolare, Carlo Capra, "Il giornalismo nell'età rivoluzionaria e napoleonica" in La stampa italiana dal 500 all' 800 (Roma-Bari, 1976), pp. 374-537; e A. Galante Garrone, "I giornali della Restaurazione, 1815-1847," in La stampa italiana del Risorgimento, (Roma-Bari, 1978), pp. 3-241. Per il giornalismo milanese in particolare, soprattutto nei suoi aspetti editoriali ed economici, cfr. M. Berengo, Intellettuali e librai nella Milano della Restaurazione (Torino, 1980).

2 Si tratta solo di un quadro panoramico e incompleto volto alla caratterizzazione e non alla documentazione della produzione periodica dell'epoca. Un'accurata appendice bibliografica sulle varie testate esaminate, accompagna i Testi $e$ Concordanze a stampa.

3 Fonte primaria d'informazione sulla tiratura di buona parte delle testate è l'Elenco delle opere stampate e pubblicate in Milano e sue Provincie, Imperial Regio Ufficio Centrale di Censura e Revisione, dal 1821 in poi. Anche il Capra, il Garrone, e soprattutto il Berengo trattano della circolazione nelle opere citate.

4 Per la censura, sia in periodo francese che austriaco, si rimanda ancora una volta agli studi citati del Capra e del Galante Garrone. Vedansi inoltre gli studi di Stuart J. Wolf, "La storia politica e sociale" e Franco Venturi, "L'Italia fuori d'Italia," in Storia d'Italia, V. III, Dal primo Settecento all'Unitì. (Torino, 1973).

5 Ancora valido l'ormai classico saggio di K.R. Greenfield, Economin \& liberalismo nel Risorgimento [Bari, 1940 (Il edizione 1964)]. Fondamentale il citato Intellettuali, del Berengo, che conferma attraverso una documentazione esemplare il cosciente impegno civile di due generazioni di intellettuali milanesi.

6 In genere ci vogliono due settimane perchè la notizia di un avvenimento europeo anche d'importanza eccezionale (quale ad esempio le vittorie o sconfitte continentali di Napoleone) venga comunicata a Parigi, Vienna o Londra, pubblicata dai giornali locali - che vengono quindi spediti a Milano - e finalmente trasmessa al lettore milanese. Il telegrafo (ottico) già usato dal Nelson a Trafalgar, viene istituzionalizzato solo verso il $\mathbf{1 8 2 5}$, e serve comunque alla trasmissione di messaggi molto brevi. Le notizie extra-europee prendono da un minimo di due mesi a un massimo di sei o otto mesi. Quanto alla compilazione di una gazzetta 
politica dallo spoglio di giornali esteri, è illuminante una corrispondenza fra il Gherardini e il Cherubini, compilatori del "Giornale italiano." Cfr. Carteggio Gherardini-Cherubini (1809-1846), Biblioteca Nazionale Braidense, Milano, Busta Autografi AC XII 31.

7 Penso in particolare ai cambiamenti di regime del 1800 e 1814 che obbligarono, per cosi dire, il redattore del "Corriere milanese" a una rapida presa di posizione.

8 Nell'Archivio di Stato di Milano, Cartella Studi, P.M. 332/333, sono raccolte alcune significative testimonianze: in periodo napoleonico, pur relativamente piu liberale, un certo Crivellari proponeva un giornale dal promettente titolo Il trombettn, "un foglio giornaliero contenente le notizie particolari di Milano."

La censura era insorta: "non sembra conveniente che un giornale abbia a trattare di materie che riguardano la pubblica Autorità, segnatamente l'arrivo, la partenza, ed alloggio de' forestieri, le cause piu clamorose e loro giudici e difensori, per le ragioni che il giornalista potrebbe essere talvolta in collisione colle viste ed operazioni dell'Autorità stessa" (Cartella 333, foglio 39). Durante la Restaurazione, basti citare il caso del Bioschi che chiedeva il permesso di pubblicare in un settimanale dal titolo La conversazione, anche" le notizie degli avvenimenti piu remarchevoli del giorno"; il commento del censore è chiaro: "tali notizie, per essere materia pericolosa e soggetta a molti inconvenienti, non se ne potrebbe senza un'estrema cautela permettere l'inserzione nei pubblici fogli" (Cartella 332, foglio 22)

9 Tutti i periodici dell'epoca, anche quelli piu specificamente letterari o di diffusione tecnico-scientifica proclamano intenzioni di rinnovamento linguistico, soprattutto nelle annuali prefazioni.

10 Alcune osservazioni sul periodico ottocentesco si trovano nell'ampio saggio di M. Dardano, Il linguaggio dei giornali italiani (Roma-Bari, 1973), pp. 7-8, che studia tuttavia il giornale odierno.

11 Gli altri generi di spettacolo, teatri meccanici, presepi animati, esibizioni di prestigiatori, spettacoli di animali, ecc. non ricevono la stessa attenzione dalla critica, anche se l'indice di gradimento dovette essere assai alto anche nelle classi piu elevate. Echi di tali spettacoli e del loro successo di pubblico sono presenti sulle pagine dei periodici in vari tipi di interventi: cronaca mondana, aneddotica, narrativa, ecc.

12 Abbondano corrispondenze da città europee anche non principali. Accanto a notizie da Parigi, Londra, Vienna, altre da Pietroburgo, Varsavia, Copenaghen, Lisbona, e Bordeaux, Majorca, Marsiglia, Cadice. . . . Dopo il 1835 compaiono su alcune testate milanesi i programmi delle stagioni teatrali dei maggiori centri europei e persino di alcune città statunitensi.

13 "Spettatore" (1818), 108.

14 "Gazzetta di Milano" $(8,2,1822)$.

151 contributi di queste pubblicazioni annuali consistevano soprattutto in rassegne delle attività svolte durante le varie stagioni. In altri casi si offrivano al lettore Ritratti "artistico-biografici" di grandi compositori, cantanti, coreografi, ecc.

16 "Censore universale dei teatri" $(3,1,1835)$.

17 "Corriere dei teatri" $(3,1,1838)$.

18 "Censore universale dei teatri $(2,5,1835)$.

19 "Censore universale dei teatri" $(4,1,1837)$. Non contente di usare materiale tradotto dai "fogli francesi" o esteri, le testate riportavano novelle, aneddoti, notizie piu o meno bizzarre, attinte anche da altri giornali italiani o addirittura milanesi.

20 "Vespa" (1827), 121.

21 "Bazar" $(3,5,1845)$

22 "Corriere delle dame" $(7,6,1837)$.

23 "Rivista europea" (1838), 345. Le discussioni sul genere appartengono però piu alla critica letteraria e musicale che al giornalismo teatrale.

24 Quando San Giovanni in Persiceto, "grossa e ricca terra del Bolognese" vien promossa al rango di città, per i festeggiamenti ufficiali si crea un apposito comitato che si procuri la presenza di "un assortimento di virtuosi piu ricercato e soprattutto di una prima donna di credito," per le indispensabili produzioni 
teatrali. I "Deputati di San Giovanni" si assicurano "un soggetto di vaglia" e il successo delle rappresentazioni è tale da attrarre un vasto pubblico di intenditori anche da Bologna. "Censore universale dei teatri" $(4,10,1837)$.

25 "Censore universale dei teatri" $(2,1,1833)$.

26 "Corriere dei teatri" $(20,7,1839)$.

27 "Corriere delle dame" $(10,1,1837)$.

28 "Gazzetta di Milano" (3,1,1833).

29 "Censore universale dei teatri" $(1,5,1835)$; ma sono espressioni comuni.

30 "Ape" (1824), 64. Nei brani che seguono si è usato il corsivo per segnalare particiolari espressioni cui si fa riferimento in seguito. (Il corsivo è mio).

31 "Censore universale dei teatri" $(3,10,1835)$.

32 "Censore universale dei teatri" $(2,1,1835)$.

33 "Corriere delle dame" $(3,1,1845)$.

34 "Vespa" (1827), 122. (Il corsivo è mio).

35 "Gazzetta di Milano" $(3,1,1833)$. (Il corsivo è mio).

36 "Poligrafo" $(15,8,1813)$. Intorno alle figure dei grandi divi della musica o dello spettacolo si vennero consolidando "industrie" ausiliarie. In seguito a questa prima attesissima visita di Paganini a Milano, ci si affretta a pubblicizzare il "ritratto, del sig. Nicolo Paganini professore di violino, disegnato dal sig. J.H. Jacob, ed inciso dal sig. Luigi Rados ... vendibile nel negozio Artaria discontro il R. Teatro alla Scala, e Ricordi nella contrada di S. Margherita, al prezzo di una lira italiana." "Corriere delle dame" $(21.11,1813)$.

37 "Almanacco del Teatro alla Scala" (1837), 48-49.

38 "Eco" $(13,3,1830)$. (Il corsivo è del testo originale).

$39 \mathrm{Sul}$ "Regime della stampa" in periodo napoleonico, e per una analisi particolare della situazione italiana si rimanda al Capra, Il giornalismo cit. pag. $485 \mathrm{e}$ seguenti. "É ben nota d'altronde la sensibilità quasi morbosa di Napoleone per gli elogi e le critiche degli scrittori..." (p. 485).

40 Compito questo che verrà assolto, con un'analisi sistematica, nell'ambito della già segnalata ricerca (Cfr. nota ${ }^{*}$ ).

41 Il Dardano vede questi limiti come esiziali allo sviluppo del genere: "poiché all'informazione pubblica mancano sia la coscienza del propro status, sia i mezzi capaci di assicurarle un'ampia diffusione, non esiste ancora una scrittura giornalistica dotata di propri caratteri." Il linguaggio, cit. p. 7. E il Dardano si riferisce qui al tardo Ottocento.

42 "Poligrafo" $(26,4,1812)$.

43 "Censore universale dej teatri" $(2,5,1835)$.

44 "Eco" (1,5,1835).

45 "Corriere delle dame" $(3,9,1845)$.

46 "Strenna teatrale europea" (1839), 192-93.

47 "Corriere delle dame" $(3,1,1845)$.

48 "Corriere delle dame" $(14,10,1804)$.

49 "Ape" (1819), 81.

50 "Censore universale dei teatri" (1,5,1833). (Il corsivo è dell'originale).

51 "Giornale italiano" $(16,4,1806)$.

52 "Corriere delle dame" $(10,1,1818)$.

53 "Pirata" $(15,5,1838)$.

54 "Corriere delle dame" $(16,4,1814)$.

55 "Pirata" $(23,7,1841)$.

56 "Almanacco del Teatro alla Scala" (1820).

57 "Gazzetta di Milano" (14,5,1842).

58 "Pirata" $(23,1,1844)$.

59 "Eco" (3,12,1830).

60 "Corriere delle dame" $(5,1,1839)$. (Il corsivo è del testo originale). 